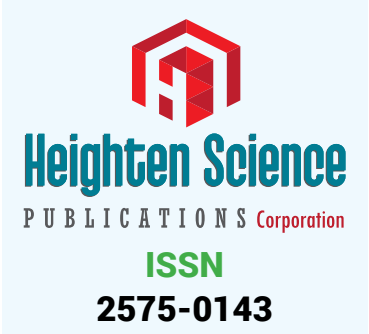

*Address for Correspondence: Macit Kalçık, Hitit University Çorum Training and Research Hospital, Çorum, Turkey, Yeniyol, Çamlık Cad. No:2, Çorum/Turkey, Fax: +903642230300 , Tel: (90)536 4921789; Email:

macitkalcik@yahoo.com

Submitted: 29 May 2017

Approved: 20 June 2017

Published: 21 June 2017

Copyright: @ 2017 Çelik O, et al. This is an open access article distributed under the Creative Commons Attribution License, which permits unrestricted use, distribution, and reproduction in any medium, provided the original work is properly cited

Keywords: Coronary artery; Aneurysm; Thrombus Pericardial effusion; Thrombolysis
Case Report

\section{Spontaneous rupture of a giant Coronary Artery Aneurysm after acute Myocardial Infarction}

\author{
Oğuzhan Çelik, Mucahit Yetim, Tolga Doğan, Lütfü Bekar, \\ Macit Kalçık* and Yusuf Karavelioğlu \\ Department of Cardiology, Hitit University Çorum Training and Research Hospital, Çorum, \\ Turkey
}

\section{CASE PRESENTATION}

Coronary artery aneurysm is commonly defined as a localized dilatation exceeding the diameter of adjacent normal coronary segments by 50\% [1]. Coronary artery aneurysms may be fusiform, involving the full circumference of the coronary artery, or saccular, involving only a portion of the circumference [2]. Causes of coronary artery aneurysms include atherosclerosis (accounting for 50\% of cases), Kawasaki disease, polyarteritis nodosa, infection, trauma, coronary dissection, percutaneous coronary angioplasty, and congenital malformations [3]. The abnormal blood flow within the coronary artery aneurysm may lead to thrombus formation, embolization, rupture, myocardial ischemia or myocardial infarction [4]. Here we present a case of a giant fusiform coronary artery aneurysm who passed away due to coronary rupture after acute myocardial infarction.

A 42 year old non-diabetic, non-hypertensive man was admitted to emergency department with chest pain continuing for 2 hours. Blood pressure and pulse rate were normal on physical examination of cardiovascular system. Electrocardiography revealed ST segment elevation in leads V1 to V4 (Figure 1). Echocardiography showed segmental wall motion abnormalities in anterior, anteroseptal and apical segments without any pericardial effusion. Laboratory analysis revealed elevated levels of Troponin I (39.6 ng/ml) and creatine kinase-myocardial band (24.5 ng/ml). He was transferred to coronary angiography unit for primary percutaneous intervention with the diagnosis of acute anteroseptal ST segment elevation myocardial infarction. Coronary angiography revealed a giant coronary artery aneurysm (20x40 mm in size) on the left anterior descending coronary artery (LAD). There was massive thrombus inside the aneurysm obstructing the distal coronary flow (Figure 2A). The circumflex and right coronary arteries were normal. Due to high thrombus burden and the lack of coronary blood flow distal to enurysm, thrombolytic therapy was administered with $10 \mathrm{mg}$ intracoronary bolus and $90 \mathrm{mg}$ intravenous infusion of tissue type plasminogen activator. A control coronary angiography after 12 hours showed partial lysis of the thrombus burden providing a TIMI III flow in the first diagonal artery. However there was TIMI 0-I flow in the distal LAD (Figure 2B and C) . The co-decision of cardiology and cardiovascular surgery team was urgent surgery. However during the surgical preperation, the haemodynamic status of the patient failed. Echocardiography revealed that massive fibrinous pericardial effusion causing cardiac tamponade (Video 1). There was no sign of free wall rupture complicating acute myocardial infarction. Immediate pericardiosentesis yielded in $750 \mathrm{cc}$ hemorrhagic fluid without any decrease in the 


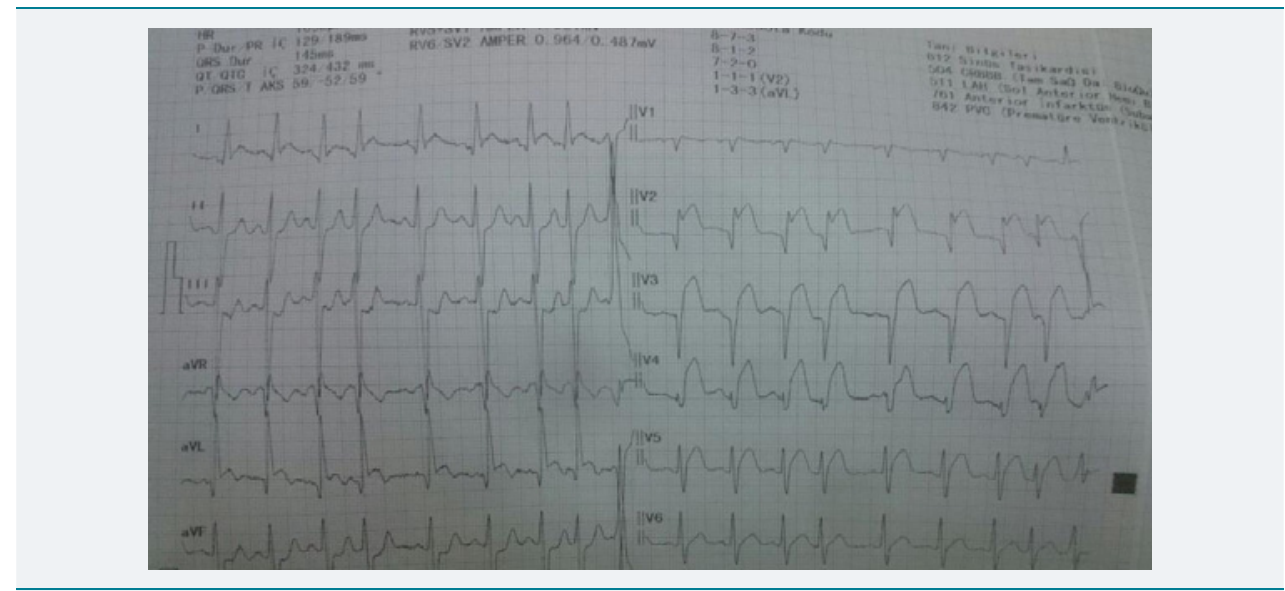

Figure 1: Electrocardiography revealing ST segment elevation in leads V1 to V4 compatible with the diagnosis of acute anteroseptal ST segment elevation myocardial infarction.

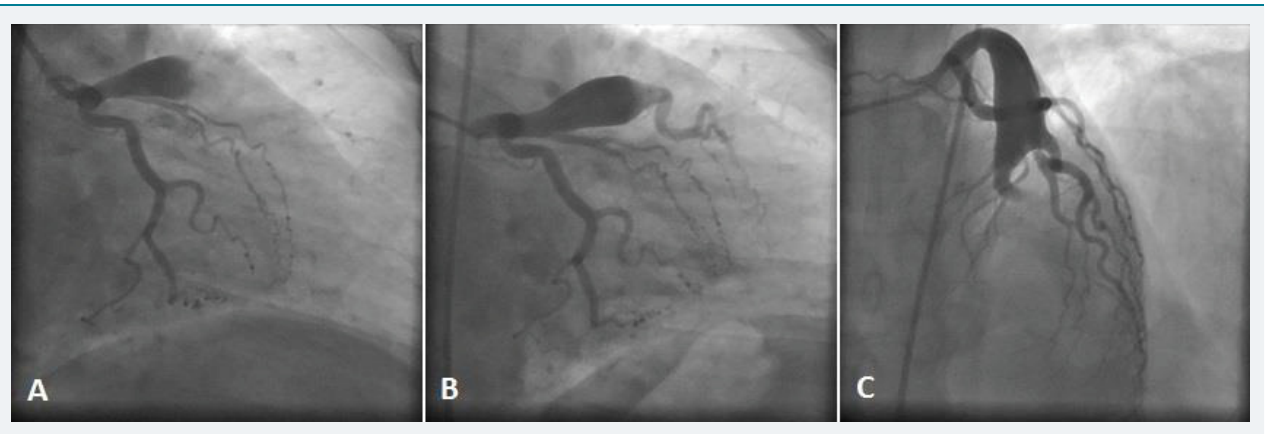

Figure 2: Coronary angiography revealed a giant coronary artery aneurysm (20x40 mm in size) on the left anterior descending coronary artery (LAD) with massive thrombus obstructing the distal coronary flow (A). Due to high thrombus burden and the lack of coronary blood flow distal to enurysm, thrombolytic therapy was performed with $10 \mathrm{mg}$ intracoronary bolus and $90 \mathrm{mg}$ intravenous infusion of tissue type plasminogen activator. A control coronary angiography 12 hours after thrombolytic therapy showed partial lysis of the thrombus burden providing a TIMI III flow in the first diagonal artery (B) and TIMI 0-I flow in the distal LAD (C).

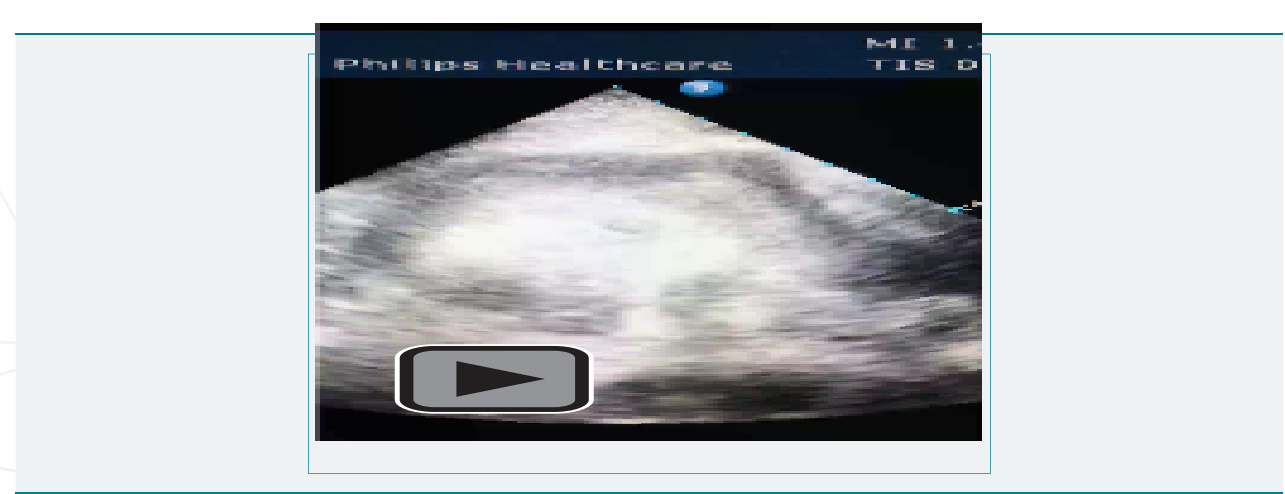

Video 1: Echocardiography revealed that massive fibrinous pericardial effusion causing cardiac tamponade.

pericardial effusion on echocardiography. Emergent chest opening by cardiovascular surgeon in the intensive care unit revealed the rupture of the giant coronary aneurysm. Soon after cardiac arrest occured before reconstruction of the LAD and the patient passed away after an unsatisfactory resusitation.

Treatment options in such a case may be thrombus aspiration, percutaneous coronary intervention with or without stenting, thrombolysis or cardiac surgery. Urgent cardiac surgery was recommended to the patient but he refused. Due to high thrombus burden and the lack of coronary blood flow distal to enurysm, we could not pass a guidewire through the aneurysm for thrombus aspiration considering the risk of coronary rupture. Therefore thrombolysis was chosen as the primary treatment option. 
Possible causes of coronary artery aneurysms include atherosclerosis, Kawasaki disease, polyarteritis nodosa, infection, trauma, dissection, and congenital malformations [3]. In the present case we did not performed autopsy to exclude Kawasaki disease or polyarteritis nodosa. However, the most probable cause was considered as the atherosclerotic coronary artery disease (accounting for $50 \%$ of all cases). Potential causes for thrombus formation in the coronary artery aneurysms include plaque rupture, the abnormal blood flow (stasis) and stenosis distal to the coronary artery aneurysm [4].

\section{REFERENCES}

1. Pahlavan PS, Niroomand F. Coronary artery aneurysm: a review. Clin Cardiol. 2006; 29: 439-443. Ref.: https://goo.gl/Fw81GM

2. Al-Khadra AH. A right coronary artery aneurysm associated with chest pain: a case report. J Family Community Med. 2009; 16: 75-76. Ref.: https://goo.gl/XaSpcs

3. Nichols L, Lagana S, Parwani A. Coronary Artery Aneurysm A Review and Hypothesis Regarding Etiology. Arch Pathol Lab Med. 2008; 132: 823-828. Ref.: https://goo.gl/RERdNT

4. Li Y, Wu C, Liu W. Coronary artery ectasia presenting with thrombus embolization and acute myocardial infarction: A case report. Medicine (Baltimore). 2017; 96: 5976. Ref.: https://goo.gl/QMLtES 